\title{
Evaluation de la contamination des eaux du fleuve Couffo dans la zone cotonnière de Djidja (Bénin) par les pesticides
}

\author{
Firmin H. AÏKPO ${ }^{1,2^{*}}$, Christophe B. CHABI ${ }^{4}$, Virgile AYI ${ }^{3}$, Luc KOUMOLOU $^{1}$, \\ Christophe S. HOUSSOU ${ }^{2}$ et Patrick A. EDORH ${ }^{1}$ \\ ${ }^{1}$ Laboratoire de Recherche en Biochimie et Toxicologie de l'Environnement (LaRBiTE), \\ Département de Biochimie et de Biologie Cellulaire, Faculté des Sciences et Techniques (FAST), \\ Université d'Abomey-Calavi (UAC), 01 BP 526 Cotonou, Bénin. \\ ${ }^{2}$ Laboratoire Pierre Pagney «Climat, Eau, Ecosystèmes et Développement » (LACEEDE), Faculté des Lettres, \\ Arts et Sciences Humaines (FLASH), Université d'Abomey-Calavi (UAC), BP 922 Cotonou, Bénin. \\ ${ }^{3}$ Laboratoire de l'Institut Régional du Génie Industriel des Biotechnologies et Sciences Appliquées \\ (IRGIB-AFRICA), 07 BP 231 Cotonou, Bénin. \\ ${ }^{4}$ Département de Biochimie et Biologie Moléculaire, Faculté de Médecine, Université de Parakou, \\ BP 123 Parakou, Bénin. \\ *Auteur correspondant, E-mail : aikpofirmin@yahoo.fr
}

\section{RESUME}

Dans la commune de Djidja, les champs de coton et autres cultures sont parfois installés non loin des berges des cours d'eau et sont souvent traités avec des pesticides. L'objectif de ce travail est d'évaluer le niveau de contamination des eaux du fleuve Couffo par les pesticides dans la commune de Djidja. Neuf (09) échantillons d'eau ont été prélevés en neuf (09) points le long du fleuve. L'analyse de ces échantillons a été faite par chromatographie en phase gazeuse après extraction et purification. Les résultats montrent une contamination des eaux par le glysophate qui varie entre 0,193 et $0,105 \mu \mathrm{g} / \mathrm{l}$, par le profénofos variant entre 0,095 et $0,128 \mu \mathrm{g} / \mathrm{l}$, par l'acétamipride qui varie entre 0,043 et $0,082 \mu \mathrm{g} / \mathrm{l}$ et par la cyperméthrine variant entre 0,096 et $0,133 \mu \mathrm{g} / \mathrm{l}$. Un programme de surveillance régulière doit être établi afin de limiter au mieux la contamination de ces eaux par les pesticides.

(C) 2015 International Formulae Group. All rights reserved.

Mots clés: Djidja, pesticides, coton, eau, contamination.

\section{INTRODUCTION}

La République du Bénin est un pays essentiellement agricole. On y cultive le coton, le maïs, le niébé, le manioc, l'igname, les cultures maraichères, etc. La protection de ces cultures contre les ravageurs au moyen des pesticides est très courante. Dans la commune de Djidja, les champs de coton et autres cultures sont parfois installés non loin des berges des cours d'eau et sont souvent traités avec des pesticides. Mais l'utilisation agricole des pesticides entraîne la contamination des ressources en eau. La pollution diffuse semble être la source dominante d'apport de pesticides vers les eaux de surface et souterraines (Gilliom et al., 2006). Les 
sources ponctuelles contribuent de façon significative à la contamination de l'eau des rivières (Reichenberger et al., 2007). Le drainage du surplus d'eau qui s'infiltre dans les sols agricoles par les canaux de drainage souterrains entraîne aussi les pesticides vers les eaux de surface (Ludvigsen, 2006). En Afrique du Sud, Fatoki et Awofolu (2003) ont rapporté des concentrations en pesticides organochlorés dans les eaux de l'East London Harbour et du fleuve Buffalo River. Mwevura (2000) avait trouvé des concentrations en résidus de pesticides organochlorés dans les eaux de la zone côtière de Dar es Salaam. Au Bénin, les eaux des réserves $\mathrm{W}$ de la Pendjari sont polluées par l'endosulfan (Soclo et al., 2003), le bassin versant du fleuve Ouémé et du lac Nokoué est contaminé par les pesticides (Pazou, 2005) et les eaux de la rivière Agbado contiennent des pesticides (Gbaguidi et al., 2011). L'objectif de ce travail est d'évaluer le niveau de contamination des eaux du fleuve Couffo par les pesticides dans la commune de Djidja.

\section{MATERIEL ET METHODES \\ Zone d'étude}

Le cadre géographique de cette recherche a été la commune de Djidja, la plus vaste des neuf (09) communes du département du Zou. Située entre $7^{\circ} 10^{\prime}$ et $7^{\circ} 40^{\prime}$ de latitude Nord, $1^{\circ} 40^{\prime}$ et $2^{\circ} 10^{\prime}$ de longitude Ouest, cette commune couvre $41,66 \%$ de la superficie totale du département. D'une superficie totale de $2184 \mathrm{~km}^{2}$, elle jouit d'un climat de type subéquatorial tendant vers le soudano-guinéen dans les parties septentrionales (Akomagni, 2006). Cette commune dispose d'une variété de types de sols (ferrallitiques, ferrugineux tropicaux, vertisols, hydromorphes) et de plaines inondables et elle est drainée par 145 $\mathrm{km}$ de cours d'eau dont le Zou et le Couffo sont les plus importants (SDS, 2004). La végétation est composée de plusieurs formations (palmeraies, savane arbustive, savane arborée, ilôts forestiers, galeries). La commune de Djidja fait partie de la zone cotonnière du Centre du Bénin. Cette zone est dominée par les céréales, les tubercules et les légumineuses. La population s'investit à $80 \%$ dans les activités agricoles.

\section{Echantillonnage}

Les sites de prélèvement des échantillons d'eau ont été retenus le long du fleuve Couffo dans les villages retenus (Zakan Kossossa, Fonkpodji et Aklinmè). Les emplacements où les prélèvements ont été effectués alimentent en eau «de boisson » les populations riveraines. Les échantillons d'eau ont été prélevés avec des bouteilles en verre ambré préalablement lavées et décontaminées. Les échantillons ont été prélevés en profondeur, en s'éloignant du fond du fleuve. Ils ont été recueillis en tenant la bouteille de prélèvement et en la plongeant dans l'eau jusqu'à ce qu'elle soit entièrement immergée, selon les procédures normalisées. Le volume d'eau prélevé par site est de 1,5 1 et constitue un échantillon composite de trois prélèvements de 0,51 par point de prélèvement et par site. Une feuille d'aluminium est placée sur le goulot afin d'empêcher tout contact entre l'échantillon et le bouchon de plastique. Ces échantillons sont codés et sont conservés dans la glace dans une glacière pendant leur transport et ensuite conservés à $4{ }^{\circ} \mathrm{C}$ jusqu'à leurs analyses. Les fiches d'identification ont été remplies.

\section{Traitement et analyse}

Tous les produits chimiques utilisés sont de qualité analytique. Les solvants d'une pureté de qualité de résidus ainsi que l'acétone, le dichlorométhane, l'hexane, le cyclohexane et le sulfate de sodium anhydre ont été obtenus auprès de Merck Co. (Darmstadt, Allemagne). L'eau utilisée est de l'eau distillée sans détergent. Une solution standard stock (entre 75 et $550 \mathrm{pg} / \mathrm{ml}$ ) a été 
préparée par pesée exacte et la dissolution a été effectuée dans l'acétone et stockée dans un congélateur à $-30{ }^{\circ} \mathrm{C}$ sans exposition à la lumière. Les solutions étalons de travail (5 $\mu \mathrm{g} / \mathrm{ml})$ ont été préparées par dilution appropriée de la solution standard stock avec le cyclohexane et conservées dans un réfrigérateur $\left(4^{\circ} \mathrm{C}\right)$.

Il a été choisi l'extraction liquideliquide pour laquelle des rendements corrects ont été rapportés pour les composés de cette recherche. La procédure d'extraction liquideliquide a été adoptée à partir de la méthode EPA 351 0C (US EPA, 1996). Un volume de $200 \mathrm{ml}$ d'échantillons d'eau a été placé dans une ampoule à décanter de capacité $250 \mathrm{ml}$. L'extraction liquide-liquide a été effectuée trois fois avec $20 \mathrm{ml}$ de dichlorométhane. Les extraits ont été combinés et séchés avec du sulfate de sodium anhydre. L'extrait résultant a été concentré à exactement $1 \mathrm{ml}$ en utilisant un évaporateur rotatif.

La détermination des pesticides a été réalisée par chromatographie en phase gazeuse. Un spectromètre de masse à haute résolution DSQII Thermo a été utilisé. Le chromatographe utilisé pour l'analyse est un chromatographe en phase gazeuse Thermo Scientific équipé d'un injecteur split/splitless et une température contrôlée Interface GCMS. Un passeur d'échantillon AS 3000 a été utilisé. Une quantité de $10 \mu \mathrm{l}$ d'aliquotes a été injectée dans le chromatographe en phase gazeuse (CPG) d'exploitation à l'aide d'une seringue avec un débit d'injection de $20 \mu 1$. La température initiale d'injection au niveau de l'injecteur a été maintenue à $70{ }^{\circ} \mathrm{C}$ pendant 5 minutes, puis augmentée et maintenue pendant 10 minutes à $310^{\circ} \mathrm{C}$, puis ramenée à $100{ }^{\circ} \mathrm{C} /$ minute. La température de la colonne au niveau du four a été maintenue à $70{ }^{\circ} \mathrm{C}$ pendant 4 minutes, puis une augmentation de $150{ }^{\circ} \mathrm{C}$ à $50{ }^{\circ} \mathrm{C} /$ minute, ensuite à $235^{\circ} \mathrm{C}$ et à $3{ }^{\circ} \mathrm{C} / \mathrm{min}$, et enfin maintenue pendant 3 minutes à $300{ }^{\circ} \mathrm{C}$ et à $50{ }^{\circ} \mathrm{C} / \mathrm{min}$. Il a été fait fonctionner le spectromètre de masse et les différentes pompes à vide afin d'atteindre un niveau de "Vacuum" stable pour l'injection. Les températures ligne de transfert, le débit du gaz (Hélium) ont été réglés. L'analyse a été effectuée avec un retard de filament multiplicateur de 5 minutes pour éviter le choc au niveau du filament d'ionisation. Les différentes valeurs présentées pour chaque site correspondent à la valeur moyenne calculée à partir des données enregistrées dans les deux essais de détermination des pesticides. Toutes les données ont été soumises à l'analyse de la variance (ANOVA) et les moyennes des concentrations en pesticides décelées ont été séparées par le test de Student $\mathrm{p}(\mathrm{T}>\mathrm{t})=0,05$.

\section{RESULTATS}

L'analyse des échantillons d'eau du fleuve Couffo prélevés dans la commune de Djidja a montré une contamination de l'eau par les pesticides (Figures 1, 2 et 3).

Dans les échantillons prélevés à Zakan Kossossa (Figure 1), le glyphosate a été détecté à des concentrations moyennes de 0,198 $\mu \mathrm{g} / \mathrm{l} \quad$ (échantillon 1), 0,205 $\mu \mathrm{g} / \mathrm{l}$ (échantillon 2) et $0,210 \mu \mathrm{g} / \mathrm{l}$ (échantillon 3). Le profénofos a été retrouvé à une concentration moyenne inférieure à la limite de détection de $0,1 \mu \mathrm{g} / \mathrm{l}$ dans l'échantillon 1 $(0,097 \mu \mathrm{g} / \mathrm{l})$, à une concentration moyenne de $0,105 \mu \mathrm{g} / \mathrm{l}$ dans l'échantillon 2 et de 0,100 $\mu \mathrm{g} / \mathrm{l}$ dans l'échantillon 3 . Les concentrations moyennes en acétamipride détectées dans tous les échantillons prélevés sont de $0,082 \mu \mathrm{g} / 1$ (échantillon 1), 0,043 $\mu \mathrm{g} / \mathrm{l}$ (échantillon 2) et $0,067 \mu \mathrm{g} / \mathrm{l}$ (échantillon 3 ) et les teneurs moyennes en cyperméthrine qui ont été mesurées sont de 0,095 $\mu \mathrm{g} / \mathrm{l}$ (échantillon 1), de $0,113 \mu \mathrm{g} / 1$ (échantillon 2) et de $0,098 \mu \mathrm{g} / \mathrm{l}$ (échantillon 3).

Les échantillons prélevés à Fonkpodji (Figure 2) présentent des concentrations moyennes de 0,215 $\mu \mathrm{g} / \mathrm{l}$ (échantillon 1), 0,221 $\mu \mathrm{g} / \mathrm{l}$ (échantillon 2) et $0,195 \mu \mathrm{g} / \mathrm{l}$ (échantillon 
3) de glyphosate. Presque absent dans les échantillons $1(0,099 \mu \mathrm{g} / \mathrm{l})$ et $2(0,095 \mu \mathrm{g} / \mathrm{l})$, le profénofos a été détecté dans l'échantillon 3 à une concentration moyenne de $0,103 \mu \mathrm{g} / \mathrm{l}$. Les concentrations moyennes en acétamipride sont de $0,063 \mu \mathrm{g} / \mathrm{l}$ (échantillon 1), 0,072 $\mu \mathrm{g} / \mathrm{l}$ (échantillon 2) et $0,058 \mu \mathrm{g} / \mathrm{l}$ (échantillon 3). Elles sont de $0,102 \mu \mathrm{g} / \mathrm{l}$ dans l'échantillon 1, de $0,096 \mu \mathrm{g} / \mathrm{l}$ dans l'échantillon 2 et de 0,101 $\mu \mathrm{g} / \mathrm{l}$ dans l'échantillon 3 pour la cyperméthrine.

Au niveau des échantillons d'Aklinmè (Figure 3), les teneurs moyennes de glyphosate sont de 0,211 $\mu \mathrm{g} / \mathrm{l}$ (échantillon 1), $0,217 \mu \mathrm{g} / \mathrm{l}$ (échantillon 2) et $0,210 \mu \mathrm{g} / \mathrm{l}$ (échantillon 3). Les teneurs moyennes en profénofos sont de $0,103 \mu \mathrm{g} / \mathrm{l}$ (échantillon 1), $0,012 \mu \mathrm{g} / \mathrm{l}$ (échantillon 2) et dans l'échantillon 3 , elles sont de $0,093 \mu \mathrm{g} / \mathrm{l}$. L'échantillon 1 a une teneur moyenne en acétamipride de 0,082 $\mu \mathrm{g} / \mathrm{l}$, celle de l'échantillon 2 est de $0,061 \mu \mathrm{g} / \mathrm{l}$ et celle de l'échantillon 3 est de $0,059 \mu \mathrm{g} / \mathrm{l}$. Ces teneurs moyennes sont de 0,107 $\mu \mathrm{g} / \mathrm{l}$ (échantillon 1), de 0,097 $\mu \mathrm{g} / \mathrm{l}$ (échantillon 2) et de $0,105 \mu \mathrm{g} / \mathrm{l}$ en ce qui concerne la cyperméthrine.

Ces figures mettent en évidence que cette série d'échantillons d'eau prélevés dans le fleuve Couffo présente une pollution par certaines molécules et traduit leur présence relative dans les différents échantillons. L'analyse de variance appliquée aux fréquences absolues des pesticides détectés au niveau des trois (03) sites révèle qu'il y a une différence très hautement significative entre les teneurs moyennes dans les eaux $(\mathrm{p}=0,000$ pour le site de Zakan Kossossa; $\mathrm{p}=0,000$ pour le site de Fonkpodji et $\mathrm{p}=0,000$ pour le site d'Aklinmè).

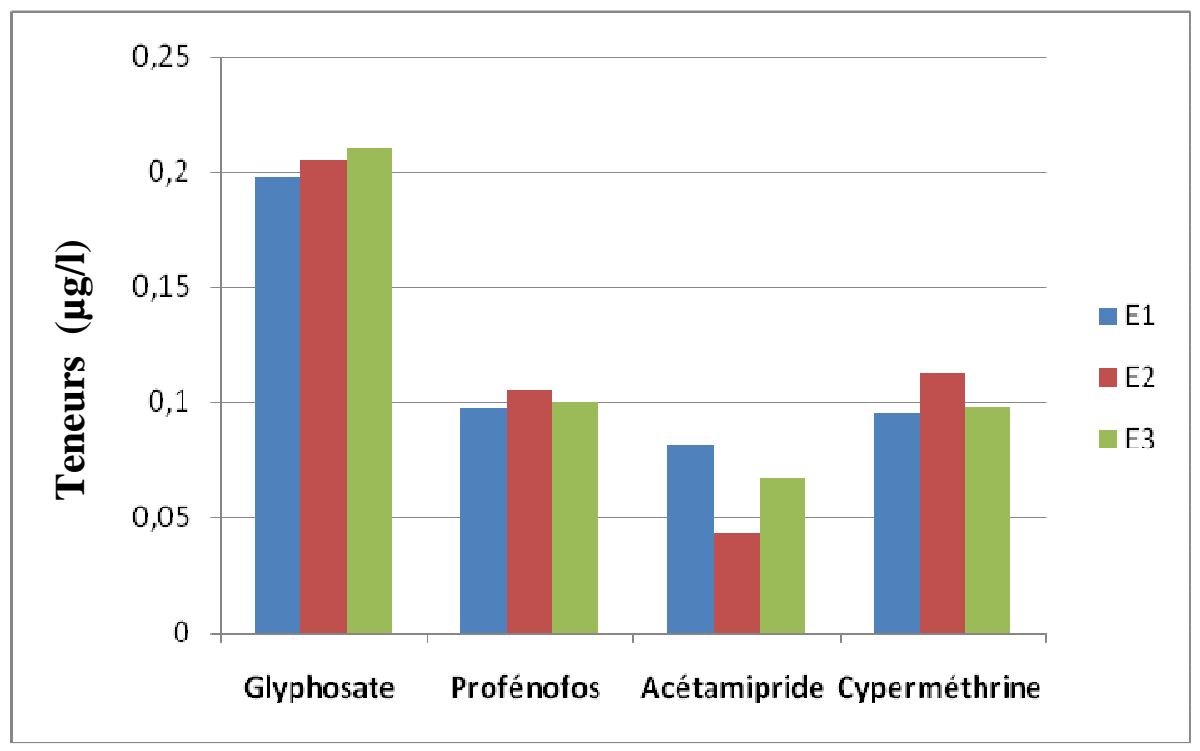

Figure 1 : Teneurs en résidus de pesticides dans les eaux du Couffo à Zakan Kossossa. E = Echantillon. 


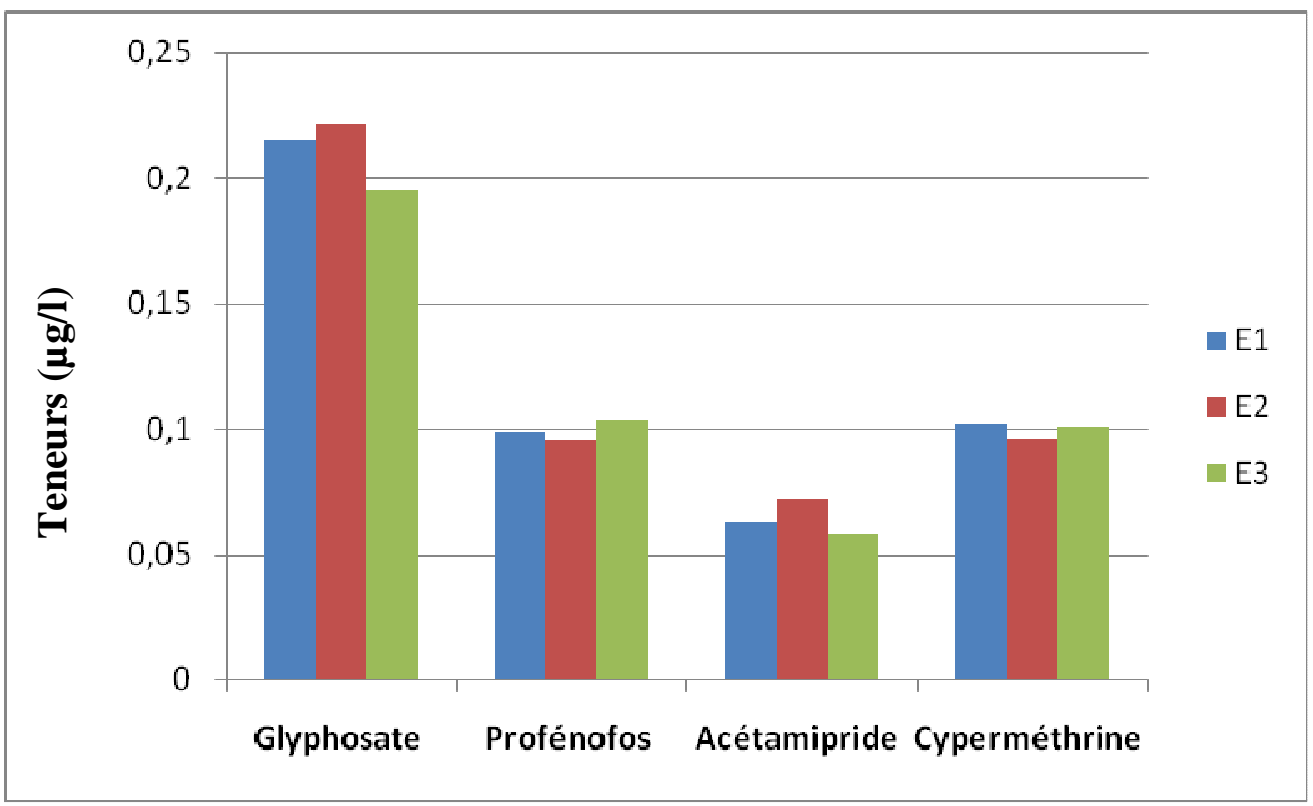

Figure 2 : Teneurs en résidus de pesticides dans les eaux du Couffo à Fonkpodji. E = Echantillon.

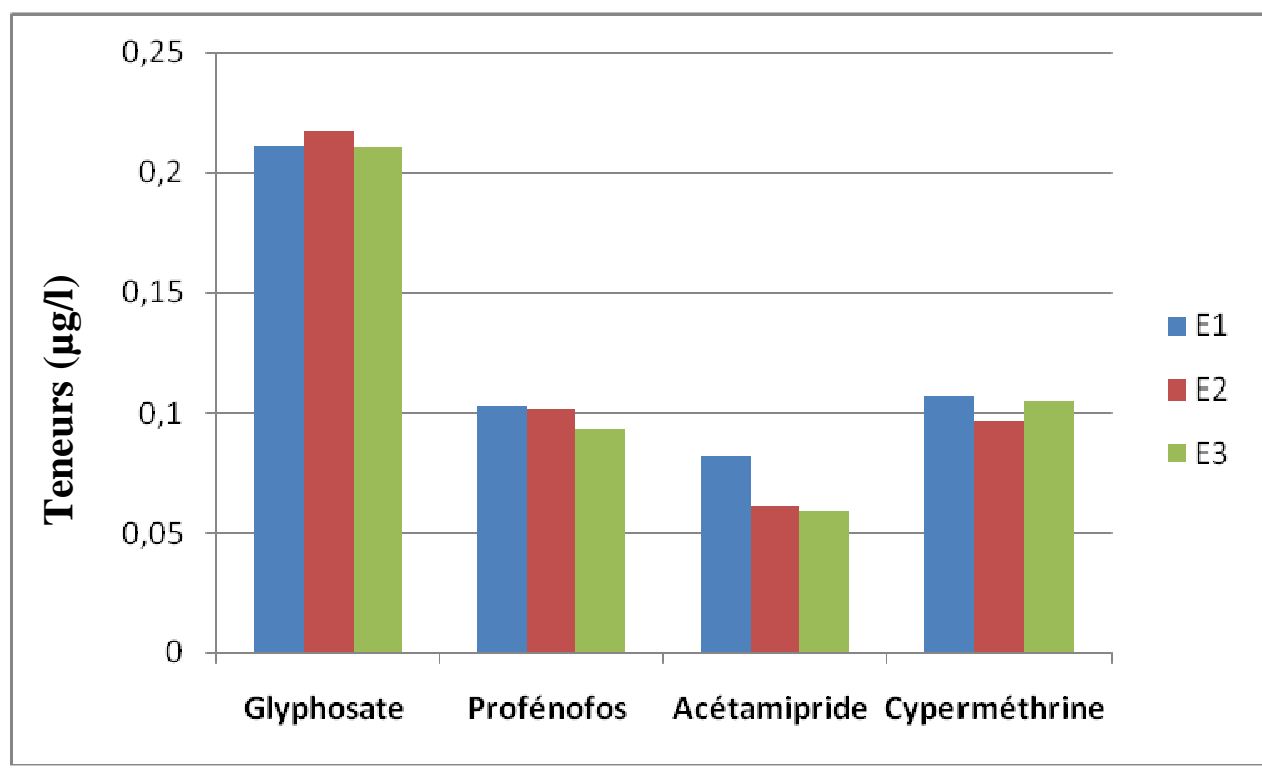

Figure 3 : Teneurs en résidus de pesticides dans les eaux du Couffo à Aklinmè. E = Echantillon. 


\section{DISCUSSION}

L'analyse des échantillons d'eau du fleuve Couffo révèle la présence des quatre pesticides (glyphosate, profénofos, acétamipride et cyperméthrine) recherchés. Le glyphosate a été détecté dans tous les échantillons d'eau à des teneurs élevées, supérieures à la norme légale recommandée pour l'eau potable par l'OMS (2004) qui est de $0,1 \mu \mathrm{g} / \mathrm{l}$ par substance individualisée. La persistance et la rémanence du produit ne peuvent expliquer à elles seules les valeurs rencontrées. Sa présence généralisée dans tous les échantillons à des teneurs élevées laisse présager d'une utilisation incontrôlée de ce produit.

Ces concentrations sont inférieures à celles trouvées dans les ruisseaux Chamilles et Marais en Suisse par Corvi et al. (2005) et à celles enregistrées aux Etats-Unis par Battaglin et al. (2005) et Coupe et al. (2011), en France par Villeneuve et al. (2011), en Hongrie par Mörtl et al. (2013) et en Norvège par Ludvigsen et Lode (2001). Mais les concentrations décelées dans les échantillons suscitent des doutes sur les risques de contamination des eaux par le glyphosate longtemps considérés comme faibles. Les teneurs moyennes en profénofos dans les échantillons 1 de Zakan Kossossa, 3 de Fonkpodji, 1 et 2 d'Aklinmè sont supérieures à la norme $(0,1 \mu \mathrm{g} / \mathrm{l})$ mais inférieures à celles décellées dans l'eau de puits en Côte-d'Ivoire par Traoré et al. (2006). L'acétamipride est décelée à des concentrations inférieures à la valeur de référence recommandée pour l'eau potable (OMS, 2004). Elles varient de 0,043 $\mu \mathrm{g} / \mathrm{l}$ à $0,083 \mu \mathrm{g} / \mathrm{l}$ pour l'ensemble des échantillons et peut confirmer les renseignements sur le devenir dans l'environnement et l'écotoxicologie de l'acétamipride contenus dans la note réglementaire REG2002-05 de l'Agence de réglementation de la lutte antiparasitaire de santé Canada (2010). En effet, cette note stipule que les produits de transformation de l'acétamipride dans l'environnement, soit IM- 1- 5 dans le sol, IM- 1- 4 dans les sédiments et IB -1-1 dans l'eau, ne devraient pas s'accumuler ou migrer dans l'environnement, ni poser de risque pour les organismes non ciblés. Les teneurs moyennes en cyperméthrine dans les échantillons 2 de Zakan Kossossa, 1 et 3 de Fonkpodji, 1 et 3 d'Aklinmè dépassent la valeur $0,1 \quad \mu \mathrm{g} / 1$ recommandée. Les caractéristiques physicochimiques influençant les risques de transfert de cette substance vers les eaux et le risque de pollution des eaux expliquent en partie les concentrations détectées qui sont supérieures à la norme.

Malgré l'échantillonnage très restreint, ces résultats concordent avec les données étrangères et plaident pour une surveillance accrue dans le pays (Bénin). Les niveaux de concentrations moyennes les plus élevées dans les échantillons, tous sites confondus, peuvent trouver encore leurs explications dans l'utilisation abusive et fréquente de ces matières actives pour le traitement des cultures. Leur détection dans les eaux, malgré leur capacité à être dégradée rapidement dans l'environnement, traduit non seulement une forte utilisation de ces produits mais également la proximité des champs aux cours d'eau.

\section{Conclusion}

Les analyses effectuées sur les eaux du Couffo révèlent de grandes disparités de contamination par les pesticides recherchés selon les sites considérés. Les résultats obtenus montrent que le niveau de contaminations des eaux est parfois supérieur à la norme relative à l'eau potable fixée par l'OMS. C'est une inquiétude grandissante, sachant que ces eaux sont consommées en grande quantité par les populations riveraines de ce fleuve. Il existe donc un risque réel d'atteinte à la santé. Ce risque sera d'autant plus élevé que les pesticides seront utilisés de plus en plus (en quantités importantes) dans la commune. Au regard des résultats obtenus et des caractéristiques physico-chimiques et toxicologiques des molécules concernées par la recherche, un programme de surveillance régulière doit être établi afin de limiter au 
mieux la contamination des eaux du fleuve par ces substances toxiques.

\section{REMERCIEMENTS}

Cette recherche a été initiée dans le cadre de la soutenance d'une thèse de Doctorat unique. Nous remercions le Laboratoire IRGIB - AFRICA qui nous a aidé pour le prélèvement, l'analyse des échantillons et l'interprétation des résultats.

\section{REFERENCES}

Agence de réglementation de la lutte antiparasitaire de santé Canada. 2010. Projet de décision d'homologation Acétamipride, Ottawa (Ontario), 80 p.

Akomagni LA. 2006. Monographie de la commune de Djidja. Programme D’Appui au Démarrage des Communes, Afrique Conseil, $44 \mathrm{p}$.

Battaglin WA, Kolpin DW, Scribner EA, Kuivila KM, Sandstrom MW. 2005. Glyphosate, other herbicides, and transformation products in Midwestern Streams, 2002. Journal of the American Water Resources Association, 41: 323 332.

Corvi C, Zimmmerli P, Ortelil D, KhimHeang S, Becker Van Sloote K. 2005. Métaux et micropolluants organiques dans les eaux, les moules et les poissons du Léman. Rapp. Comm. Int. Prot. Eaux Léman Contre pollut., Compagne 2004: $55-78$.

Coupe RH, Kalkhoff SK, Capel PD, Gregorie C. 2011. Fate and transport of glyphosate and aminomethylphosphonic acid in surface waters of agricultural basin. Pesticides Management Science, 67: doi : 10.1002/PS 2212.

Fatoki OS Awofolu RO. 2003. Methods for selective determination of persistent organochlorine pesticide residues in water and sediments by capillary gas chromatography and electron - capture detection. Jounal of chrmatography $A$. 983: $225-236$.

Gbaguidi M A N, Soclo H H, Issa Y M, Fayomi B, Dognon R, Agagbé A, Bonou
C, Youssao A, Dovonou LF Sanni A. 2011. Evaluation quantitative des résidus de pyréthrinoïdes, d'aminosphosphate et de triazines en zones de production de coton au Bénin par la méthode ELISA en phase liquide : cas des eaux de la rivière Agbado. Int. J. Biol. Chem. Sci., 5(4): 1476 - 1490 .

Gilliom RJ, Marcogliese DJ, Barbeau S. 2006. The quality of our Nation's Waters Pesticides in the Nation's Streams and Ground water 1992-2001. US Geological Survery Circular 1291.

Ludvigsen GH. 2006. The Agricultural Environmental Monitoring Program in Nordic Workshop on pesticide monitoring in the environment. UPPsala, $78-82$.

Ludvigsen GH, Lode O. 2001. Results from the agricultural and environmental monitoring program of pesticides in Norway 1995-1999. Fresenius Environmental Bulletin, 10: 470 - 474.

Mörtl M, Németh G, Juracsek J, Darvas B, Kamp L, Rubio F, Székas A. 2013. Determination of Glyphosate residues in Hungarian water samples by immunoassay, Microchemical Journal, 107: 143-151.

Mwevura H. 2000. Study on the levels of organochlorine pesticide residues from selectedd aquatic bodies of Tanzania. Tanzania, University of Dar es Salaam.

OMS (Organisation Mondiale de la Santé). 2004. Directives de Qualité pour l'Eau de Boisson ( $3^{\mathrm{e}}$ edn, vol 1). OMS. Genève, Suisse ; $110 \mathrm{p}$.

Pazou E. 2005. Les résidus de pesticides chimiques de synthèse dans les eaux, les sédiments et les espèces aquatiques du bassin versant du fleuve Ouémé et du Lake Nokoué. Thèse de Doctorat, FLASH/UAC, Bénin, 217 p.

Reichenberger SM, Bach A, Skitschak A, Frede HG. 2007. Mitigation strategies to reduce pesticides input into ground and surface water and their effectiveness, Sci. Total. Environ., 38(4): 1 -35. 
SDS (Schéma de Développement Sectoriel). 2004. Document de synthèse, Diagnostic, Vision et Planification de projets sur 2004 - 2008 du secteur de l'agriculture, de l'élevage et de l'exploitation des ressources naturelles dans la commune de Djidja, PADeCOM/Zou, 159 p.

Soclo HH, Azontondé AH, Dovonon LF, Djibril R, Sagbo AU. 2003. Etude de l'impact de l'utilisation des engrais chimiques et des pesticides par les populations riveraines sur les écosystèmes (eau de surface, substrats des réserves de faune) dans les complexes des aires protégées de la Pendjari et du $\mathrm{W}$ du Bénin. Rapport final, MAEP, CENAGREF, Cotonou, Bénin, 135 p.

Traoré SK, Mamadou K, Dembele A, Lafrance P, Houénou P. 2006.
Contamination de l'eau souterraine par des pesticides en régions agricoles en Côte -d'Ivoire (centre Sud et Sud-ouest). Journal Africain des Sciences de l'Environnement, 1(1): 1 - 9.

US EPA (United State's Environmental Protection Agency). 1996. Methode 351 oc, Separatory Funnel Liquid - Liquid Extraction (Revision 3 - December, 1996) 846 CH4. 2 - 1.

Villeneuve A, Larroudé S, Hubert JF. 2011. Herbicide contamination of freshwater ecosystems: Impact on Microbial Communities. In Pesticides: Formulations, Effects, Fate, Stoytcheva M (ed.). HAL, InTech Open: France; 285-312. HAL Id: bioemco-00567203 